\title{
Eosinofilia en esputo versus óxido nítrico en aire exhalado: aplicación clínica en el asma
}

\section{Eosinophils in induced sputum versus nitric oxide in exhaled air: clinical utility in bronchial asthma}

\author{
M.J. Álvarez ${ }^{1}$, P. Uribe ${ }^{1}$, A. Echegoyen ${ }^{2}$, E. Almudévar ${ }^{2}$, J.M. Olaguíbel ${ }^{1}$, E. Urbiola ${ }^{2}$
}

\section{RESUMEN}

Fundamento. La eosinofilia en esputo inducido permite optimizar el tratamiento antiinflamatorio en el asma pero sus requerimientos técnicos hacen poco viable su aplicación clínica. Evaluamos si el óxido nítrico $\left(\mathrm{FE}_{\mathrm{NO}}\right)$, un marcador inflamatorio de fácil medición, podía suplir a la eosinofilia en esputo, así como la relación que ambos índices mostraban con aspectos clínicos y funcionales del asma.

Métodos. Se estudiaron 106 asmáticos (50 con tratamiento antiinflamatorio $[\mathrm{AB}+]$ y 56 sin él $[\mathrm{AB}-])$ y 15 controles. Tras completar un cuestionario clínico, se les realizó medición de $\mathrm{FE}_{\mathrm{No}}$, espirometría forzada e inducción de esputo. Se calculó el valor de pendiente de la curva concentración-respuesta (CCR) obtenida tras la inhalación de salino hipertónico.

Resultados. Los resultados de $\mathrm{FE}_{\mathrm{NO}}$ y de eosinofilia en esputo fueron valorables en $100 \%$ y $81 \%$ de los casos respectivamente. Los valores de $\mathrm{FE}_{\mathrm{NO}}$ fueron superiores en $\mathrm{AB}$ - que en $\mathrm{AB}+\mathrm{y}$ en éstos respecto a controles. $\mathrm{La}$ eosinofilia, mayor en asmáticos que en controles aunque sin diferencias entre $\mathrm{AB}+\mathrm{y} \mathrm{AB}-$, se correlacionó con la $\mathrm{FE}_{\mathrm{NO}}$ y se comportó de forma paralela a la pendiente CCR. Había correlación entre el nivel de control del asma y los índices inflamatorios (eosinofilia y $\mathrm{FE}_{\mathrm{NO}}$ ) en el grupo $\mathrm{AB}$ - pero no en el $\mathrm{AB}+$.

Conclusión. La obtención de $\mathrm{FE}_{\mathrm{NO}}$ es rápida, sencilla y da resultados inmediatos que se correlacionan con la actividad del asma. Puede desempeñar un papel relevante en el diagnóstico y manejo terapéutico inicial de asma, aunque se necesitan más estudios para evaluar su aplicación en la monitorización a largo plazo de la enfermedad, por la influencia tan notable que tiene sobre sus niveles el tratamiento antiinflamatorio.

Palabras clave. Asma. Esputo inducido. Eosinófilos. Óxido nítrico. Agonistas osmóticos.

\section{An. Sist. Sanit. Navar. 2009; 32 (2): 217-225}

1. Servicio de Alergología. Hospital Virgen del Camino. Pamplona.

2. Servicio de Anatomía Patológica. Hospital Virgen del Camino. Pamplona.

Recepción: 25 de febrero de 2009

Aceptación provisional: 27 de abril de 2009

Aceptación definitiva: 24 de junio de 2009

\begin{abstract}
Background. Assessment of eosinophils in induced sputum can help to optimize anti-inflammatory therapy in bronchial asthma, but this is a very demanding technique. The aim of the study is to compare nitric oxide in exhaled air (FeNO), an easy to measure inflammatory biomarker, with eosinophils in sputum in terms of relationship with clinical and functional parameters.
\end{abstract}

Methods. 106 asthmatic patients (50 in anti-inflammatory therapy $[\mathrm{AB}+]$ and 56 without it $[\mathrm{AB}-])$ and 15 controls were included. After filling a clinical questionnaire, FeNO measurement, forced spirometry and sputum induction by bronchial challenge with hypertonic saline solution were performed.

Results. Adequate measurements of FeNO and eosinophils were obtained in $100 \%$ and $81 \%$ of the patients, respectively. $\mathrm{FE}_{\mathrm{NO} \text { w }}$ were higher for $\mathrm{AB}$ - compared to $\mathrm{AB}+$ and controls. The percentage of eosinophils in sputum was higher in asthmatic patients compared to controls but without differences between both asthmatics groups and was well correlated with the slope of the dose-response curve of bronchial challenge. In the ABgroup, FeNO and eosinophils were well correlated with asthma control level.

Conclusion. $\mathrm{FE}_{\mathrm{NO}}$ measurement is readily available and well correlated with clinical and functional markers asthma expression. Anti-inflammatory therapy blunts FeNO levels compromising its utility in the long-term follow-up of asthma patients.

Key words. Asthma. Induced sputum. Eosinophils. Nitric oxide. Osmotic agonists. 


\section{INTRODUCCIÓN}

El asma es una enfermedad inflamatoria crónica de las vías respiratorias caracterizada por hiperreactividad bronquial (HRB) y obstrucción más o menos reversible al flujo aéreo ${ }^{1}$. Los objetivos que debe perseguir el tratamiento del asma deben dirigirse tanto a la reducción del deterioro o impairment (síntomas, necesidad de medicación de rescate, calidad de vida...), como del riesgo (descenso de la función pulmonar, efectos secundarios de la medicación...) debidos a la enfermedad ${ }^{1}$. A pesar de que actualmente disponemos de potentes fármacos antiinflamatorios, los estudios epidemiológicos muestran reiteradamente que en todo el mundo, el asma continúa influyendo negativamente en la vida de los pacientes (exacerbaciones, atenciones urgentes, ausencias laborales/escolares) ${ }^{2}$, sin que se cumplan los objetivos que recomiendan las guías ${ }^{1,3}$. Los índices empleados en el diagnóstico y monitorización del asma, son los síntomas clínicos, a menudo inespecíficos ${ }^{4} \mathrm{y}$ la función pulmonar basal (espirometría forzada) que en el asma levemoderada suele ser normal. Esta ausencia de parámetros objetivos conduciría a situaciones indeseables de infra o supratratamiento y podría ser al menos una de las causas del mal control del asma.

Se impone por lo tanto la necesidad de disponer de índices objetivos que nos permitan mejorar el control del asma y optimizar su tratamiento. Lógicamente y dado su carácter inflamatorio, sería deseable contar con un parámetro que midiera de forma no invasiva la inflamación de las vías respiratorias. Para ello, en la década de 1990 se rescató el análisis del esputo, en desuso debido a la dificultad de obtener muestras adecuadas en asmáticos y al escaso rendimiento de su análisis. La inhalación de salino hipertónico y el pretratamiento de la muestra con el mucolítico ditiotreitol, mejoraron radicalmente la rentabilidad del procedimiento, permitiendo estandarizar la técnica de inducción de esputo ${ }^{5-7}$. Se constató que la eosinofilia en esputo permitía diferenciar a asmáticos de individuos sanos o con otras enfermedades respira- torias (infecciones, EPOC, rinitis alérgica, etc. $)^{8}$ y que además, se modificaba de forma paralela a los cambios en la exposición alergénica $^{8,9}$ y en el control clínico de la enfermedad $^{10,11}$, lo que permitió establecer límites de normalidad en sus valores ${ }^{12}$. Actualmente se considera que la técnica aporta claras ventajas ya que además de predecir la respuesta al tratamiento antiinflamatorio ${ }^{13}$, permite optimizar éste, consiguiendo con la misma dosis acumulada, reducir el número de exacerbaciones de asma ${ }^{10}$. No obstante, los condicionamientos de la técnica -personal especializado, capacidad de expectoración del paciente, requerimiento de tiempo e imposibilidad de obtener resultados inmediatos entre otros-, dificultan su aplicación a la evaluación rutinaria del paciente asmático.

$\mathrm{El}$ óxido nítrico $\left(\mathrm{FE}_{\mathrm{NO}}\right)$, fácil y rápidamente cuantificable en el aire exhalado mediante quimioluminiscencia, se eleva en el asma así como en otras enfermedades respiratorias que cursan con inflamación ${ }^{14}$. Los valores de $\mathrm{FE}_{\mathrm{NO}}$ se modifican paralelamente a la exposición alergénica ${ }^{15}$, se elevan en las exacerbaciones del asma ${ }^{16}$ y se reducen rápidamente tras la administración de tratamiento esteroideo ${ }^{17}$. La cuantificación de $\mathrm{FE}_{\mathrm{NO}}$ es rápida, sencilla y requiere una mínima colaboración por el paciente, lo que unido a la correlación de sus valores con la eosinofilia en esputo ${ }^{11}$, sugiere que este índice podría desempeñar un papel relevante en el diagnóstico y seguimiento del paciente con asma.

Nuestra hipótesis fue evaluar si en los pacientes asmáticos, la información obtenida con los valores de $\mathrm{FE}_{\mathrm{NO}}$ era equiparable a la que aportaba la eosinofilia en esputo y estudiar como ambos índices se relacionaban con los indicadores clínicos y funcionales de asma.

\section{PACIENTES Y MÉTODOS}

Se seleccionaron los 106 pacientes con asma que acudieron sucesivamente a la consulta de Alergología. De ellos, 50 seguían tratamiento con corticoides inhalados (200 $\mu \mathrm{g}$ fluticasona o equivalente) 
mientras que 56 sólo empleaban broncodilatadores -adrenérgicos de corta duración a demanda. Como grupo control se incluyeron 15 pacientes no atópicos, sin síntomas respiratorios y valores espirométricos normales, con la finalidad exclusiva de tener un valor de referencia del nivel del $\mathrm{FE}_{\mathrm{NO}}$ en individuos sanos. Los controles eran voluntarios, miembros del servicio de Alergología, o estudiantes de medicina. Los datos clínicos se muestran en la tabla 1 . Los suje- tos asmáticos sin tratamiento antiinflamatorio (AB-) como era esperable debido a las características de la historia natural de la enfermedad eran más jóvenes y con mayor frecuencia varones (el asma en adultos y adolescentes suele ser más grave en mujeres), en comparación con los pacientes en tratamiento antiinflamatorio de fondo $(\mathrm{AB}+)$. No obstante, no parece que existan influencias de la edad o el sexo en los niveles de $\mathrm{FE}_{\mathrm{NO}}$ o eosinófilos en esputo.

Tabla 1. Datos clínicos de los pacientes incluidos en el estudio.

\begin{tabular}{lccc}
\hline & Controles & BD a demanda & Corticoides inhalados \\
\hline $\mathbf{N}$ & 15 & 56 & 50 \\
Sexo (\%mujeres) & $62 \%$ & $48 \%$ & $68 \%$ \\
Edad (a) & $42(20-55)$ & $26(12-48)$ & $38(14-58)$ \\
Evolución Asma (a) & - & $6(2-35)$ & $11(1-41)$ \\
Atopia (\%) & - & $82 \%$ & $46 \%$ \\
\hline
\end{tabular}

\section{Protocolo}

El estudio se realizó en un único día en que los sujetos cumplimentaron el cuestionario de síntomas de asma ACT (Asthma Control Test $)^{18}$. Se procedió después a la cuantificación de $\mathrm{FE}_{\mathrm{NO}}$, a la realización de una espirometría forzada $\left(\mathrm{FEV}_{1}\right.$ y de $\mathrm{FEV}_{1} /$ FVC), y por último, a la obtención de una muestra de esputo inducido. Este protocolo siguió normas de práctica clínica habitual, cumplía las normas éticas de la Comisión de Investigación del hospital Virgen del Camino, y la Declaración de Helsinki.

\section{Cuestionario ACT}

El cuestionario ACT, recientemente validado al español ${ }^{18}$, consta de 5 preguntas referidas a las últimas 4 semanas en las que el paciente gradúa de 1 a 5 el impedimento para realizar actividades cotidianas, la frecuencia de síntomas de disnea y de asma, la necesidad de medicación de rescate y valora subjetivamente el control de su asma. La puntuación final oscila entre 5 (asintomático) y 25 puntos (síntomas máximos).

\section{Cuantificación de $\mathrm{FE}_{\mathrm{No}}$}

Se realizó mediante quimioluminiscencia con un analizador portátil (NioxMiNo, Aerocrine AB; Smidesvägen, Suecia). El paciente, partiendo de volumen residual al que llegaba mediante una espiración lenta, inhalaba aire del dispositivo hasta capacidad pulmonar total, procediendo después a expulsarlo lentamente manteniendo un flujo constante de $50 \mathrm{~L} / \mathrm{min}$ durante un mínimo de 6 segundos.

\section{Inducción de esputo}

Para la inducción de esputo ${ }^{8,9}$ se administró suero salino al $5 \%$ con un nebulizador ultrasónico (Ultraneb 2000, De Vilbiss, Somerset PA, USA), durante tres periodos de 10 minutos. Previo al inicio de la prueba y después de cada periodo, se midieron los valores de $\mathrm{FEV}_{1}$, instándose al paciente a expectorar. Se empleó el cociente $\mathrm{FEV}_{1} / \mathrm{FVC}$ como marcador de obstrucción bronquial. A partir del registro de $\mathrm{FEV}_{1}$ se obtuvo una curva concentración-respuesta, cuya pendiente se calculó como el cociente entre la 
concentración de salino inhalada (5\%) y la máxima caída objetivada de $\mathrm{FEV}_{1}$.

A la muestra de esputo se le añadió un volumen igual de ditiotreitol (Sputasol, Unipath LTD. Basingstoke, Hampshire, UK) al 1/100 y se mezcló a temperatura ambiente durante 15 minutos. Se pasó la solución a través de un filtro de $0,42 \mu \mathrm{m}$ de diámetro (Millipore, Somerset, PA, USA) y se centrifugó durante 10 minutos a $1.500 \mathrm{rpm}$. Una vez retirado el sobrenadante, el "pellet" se resuspendió en salino al $0,9 \%$, y se procedió, tras la realización de tinciones Papanicolau y Giemsa, al recuento diferencial de células. La muestra era adecuada cuando la contaminación de células escamosas era $<20 \%$.

\section{Estadística}

Se empleó el programa SPSS 16.0 para realizar el análisis estadístico, Los resultados se expresaron como medianas y recorrido intercuartílico (IQR). Se emplearon los tests de Mann-Whitney y de Spearman para evaluar las diferencias entre grupos y las correlaciones entre variables, respectivamente. Se consideraron significativos los valores de $\mathrm{p}<0,05$.

\section{RESULTADOS}

Todos los sujetos cumplimentaron el cuestionario de síntomas ACT y se les realizó una espirometría forzada y una cuantificación de $\mathrm{FE}_{\mathrm{NO}}$. En 81 pacientes se procedió a la inducción de esputo, obteniéndose muestras adecuadas para el recuento de eosinófilos en 66 de ellos (81\%). Los resultados obtenidos en los grupos de pacientes se muestran en la tabla 2. Los valores de $\mathrm{FE}_{\mathrm{NO}}$, adecuados para su valoración en todos los sujetos, eran superiores en asmáticos y dentro de este grupo, entre los que no recibían tratamiento con corticoides respecto a los tratados $(\mathrm{p}<0,05)$. No se objetivaron diferencias en los restantes índices estudiados.

Tabla 2. Resultados del estudio obtenidos en los grupos de pacientes.

\begin{tabular}{lccc}
\hline & Controles & BD a demanda & Corticoides inhalados \\
\hline FEV $_{1}$ (\%) & $104(97-112)$ & $104(96-116)$ & $101(82-118)$ \\
Pendiente salino & NR & $0,43(0,15-1,47)$ & $0,40(0,15-1,86)$ \\
Puntuación ACT & NR & $23(19-25)$ & $23(19,7-25)$ \\
FE $_{\text {No }}$ (ppb) & $14,4(9,5-18)^{*}$ & $46(23,25-88,75)^{*}$ & $33(24,5-66,0)^{*}$ \\
Eosinófilos (\%) & NR & $5(2-20)$ & $5(2-10)$ \\
\hline
\end{tabular}

Valores de mediana e recorrido intercuartílico (IQR). ACT: Cuestionario "Asthma Control Test"; $\mathrm{FE}_{\mathrm{NO}}$ (ppb): fracción exhalada de óxido nítrico en partes por billón. Pendiente salino: pendiente de la curva concentración-respuesta realizada tras la inhalación de salino hipertónico. NR: no realizado. * $\mathrm{p}<0,05$.

La eosinofilia en esputo y los valores de $\mathrm{FE}_{\mathrm{NO}}$ se asociaban entre sí (Fig. 1), así como con los indicadores de síntomas y de HRB, si bien los coeficientes de correlación eran muy discretos. Sólo la eosinofilia, y no los valores de $\mathrm{FE}_{\mathrm{NO}}$, lo hacía con el grado de obstrucción basal de la vía respiratoria (Tabla 3), aunque también de forma discreta. Al analizar por separado los grupos de asmáticos, se observó que las correlaciones de los indicadores de síntomas, tanto con la eosinofilia en esputo (Fig. 2) como con los valores de $\mathrm{FE}_{\mathrm{NO}}$ (Fig. 3) se incrementaban ligeramente en el grupo de asmáticos que no recibía tratamiento antiinflamatorio (AB-)(manteniéndose no obstante en valores de correlación discretos), mientras que se perdía la significación estadística en grupo $(\mathrm{AB}+)$ manteniéndose para el $\mathrm{FE}_{\mathrm{NO}}$ solo una tendencia que tampoco llegó a ser significativa. 


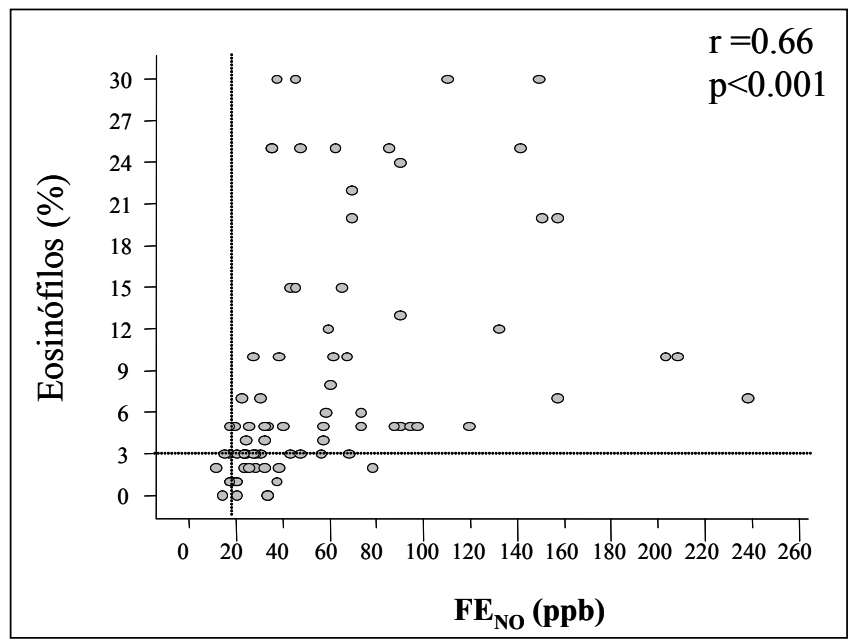

Figura 1. Correlación entre la eosinofilia en esputo y los valores de $\mathrm{FE}_{\mathrm{NO}}$ en la muestra completa de pacientes.

Tabla 3. Correlaciones entre las variables en el total de la muestra de pacientes.

\begin{tabular}{|c|c|c|c|c|c|}
\hline & ACT & $\mathrm{FEV}_{1} / \mathrm{FVC}$ & P. salino & Eosinófilos & $\mathrm{FE}_{\mathrm{No}}$ \\
\hline ACT & // & NS & $\mathrm{R}=0,45$ ** & $\mathrm{R}=-0,34$ ** & $\mathrm{R}=-0,41$ * * \\
\hline $\mathrm{FEV}_{1} / \mathrm{FVC}$ & NS & // & NS & $\mathrm{R}=-0,37$ * * & NS \\
\hline P. salino & $\mathrm{R}=0,45$ * * & NS & // & $\mathrm{R}=-0,28$ * & $\mathrm{R}=-0,445^{* *}$ \\
\hline Eosinófilos & $\mathrm{R}=-0,34$ * * & $\mathrm{R}=-0.37$ * * & $\mathrm{R}=-0,28$ * & // & $\mathrm{R}=0,66$ * * \\
\hline $\mathrm{FE}_{\mathrm{No}}$ & $\mathrm{R}=-0,41$ * * & NS & $\mathrm{R}=-0,445^{* *}$ & $\mathrm{R}=0,66$ * * & // \\
\hline
\end{tabular}

Abreviaturas: ACT: Cuestionario "Asthma Control Test"; FENO (ppb): fracción exhalada de óxido nítrico en partes por billón. P. salino: pendiente de la curva concentración-respuesta realizada tras la inhalación de salino hipertónico. NS: no significativo. ${ }^{*} \mathrm{p}<0,05 ;{ }^{*}$ : $\mathrm{p}<0,01$

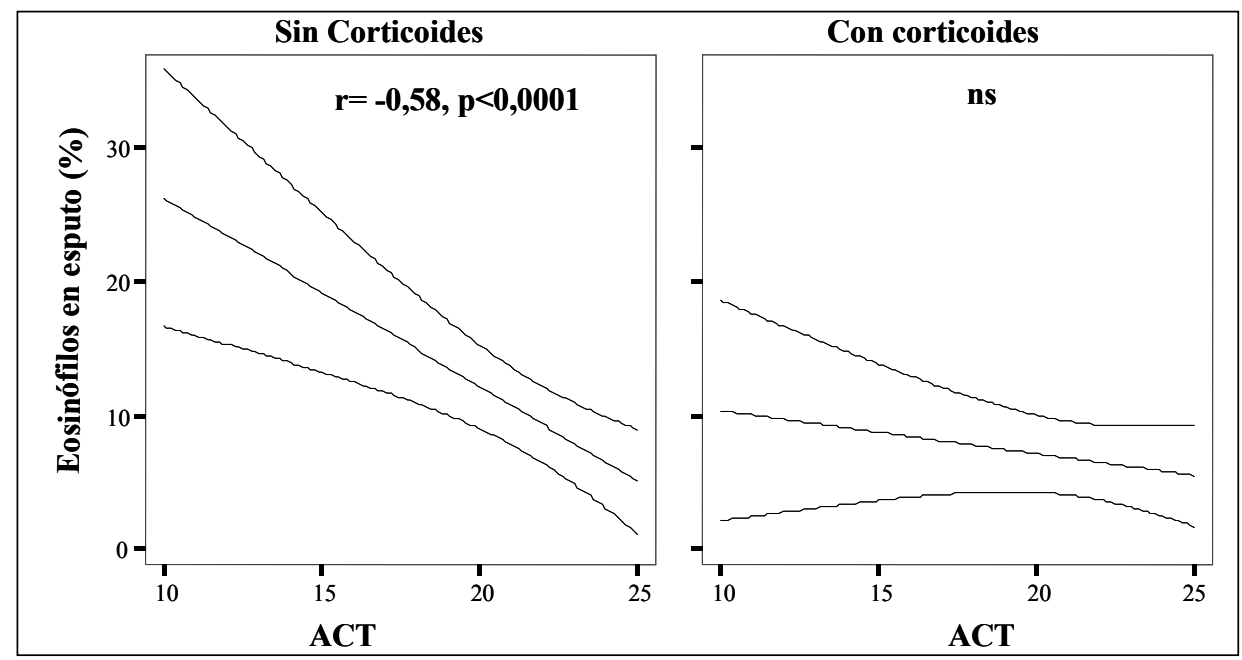

Figura 2. Correlación entre la eosinofilia en esputo y la puntuación del cuestionario ACT en los pacientes agrupados en función de su tratamiento con o sin corticoides inhalados. 


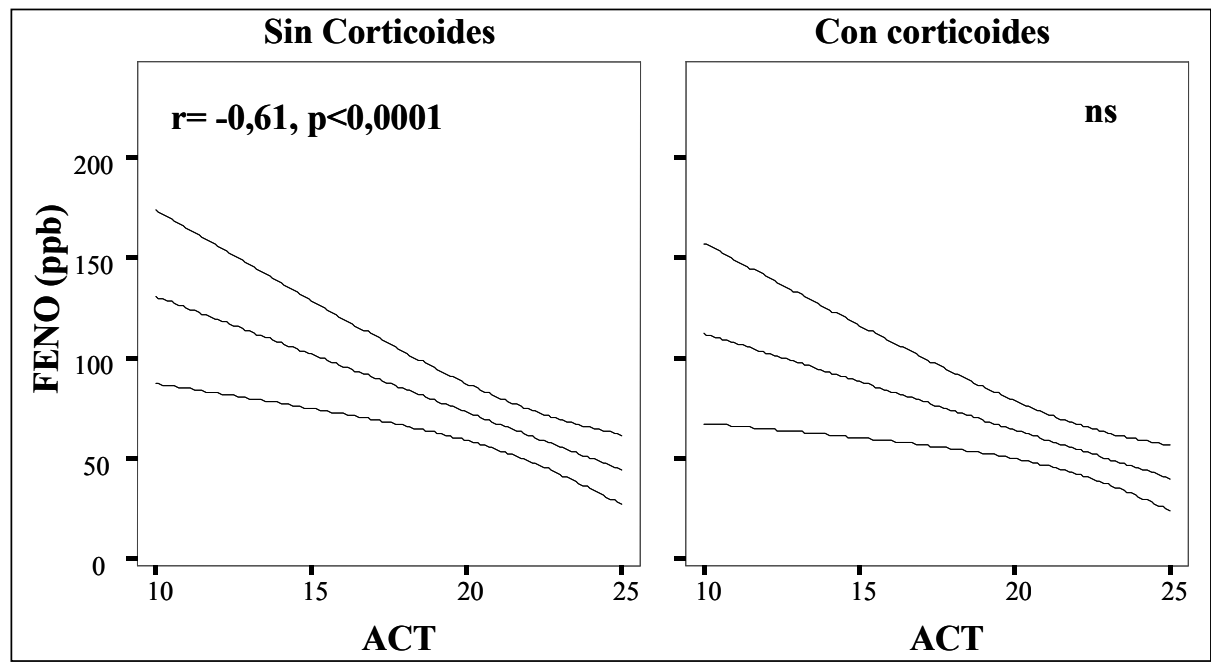

Figura 3. Correlación entre los valores de $\mathrm{FE}_{\mathrm{NO}}$ y la puntuación del cuestionario ACT en los pacientes agrupados en función de su tratamiento con o sin corticoides inhalados.

\section{DISCUSIÓN}

En contraste con la medición de $\mathrm{FE}_{\mathrm{NO}}$, que fue posible en la totalidad de los individuos, sólo fueron aptas para el análisis $81 \%$ de las muestras de esputo inducido, lo que está en consonancia con la rentabilidad entre el $60 \%$ y el $100 \%$ que la literatura cita para la técnica ${ }^{19}$. Como ha sido previamente descrito ${ }^{20}$ los valores de $\mathrm{FE}_{\mathrm{NO}}$ se correlacionaban con la eosinofilia en esputo y eran superiores en asmáticos que en controles. Además, se apreciaban valores de $\mathrm{FE}_{\mathrm{NO}}$ más altos en los asmáticos que no llevaban tratamiento esteroideo, lo que está de acuerdo con la elevada sensibilidad de este marcador al tratamiento antiinflamatorio $^{15,21,22}$. Sin embargo, esta diferencia no se podía apreciar en la eosinofilia en esputo cuyos valores, por encima del límite de normalidad establecido en $2 \%{ }^{12}$, no diferían en los grupos de asmáticos en función de su tratamiento de base.

La hiperreactividad bronquial, o respuesta exagerada de las vías respiratorias a diversos estímulos, es una característica central del asma. Los estímulos directos actúan contrayendo el músculo liso bronquial mientras que los estímulos indirectos inducen broncoconstricción a través de la liberación de mediadores considerándose, la respuesta bronquial a ellos un indicador del grado de inflamación y por ende, de la actividad del asma ${ }^{23,24}$. Los estímulos osmolares son broncoconstrictores indirectos; entre ellos se cuenta el salino hipertónico cuyo efecto se cuantifica a través de los valores de $\mathrm{PC}_{20}$ (concentración que induce una caída de $\mathrm{FEV}_{1}$ del $20 \%$ ) o de la construcción de curvas concentraciónrespuesta (CCR) obtenidas en las pruebas de exposición inhalada. Nuestro diseño de estudio, realizado a volumen corriente y con una única concentración de salino, sólo nos permitió calcular la pendiente de la CCR, cuyos valores se asociaban a los de inflamación bronquial, dada tanto por la eosinofilia como por los valores de $\mathrm{FE}_{\mathrm{NO}}$. No obstante, al igual que ocurría con la eosinofilia en esputo, la HRB a salino hipertónico no difería entre los asmáticos que seguían tratamiento con corticosteroides inhalados y los que sólo recibían broncodilatadores a demanda. Estas diferencias pueden ser consecuencia tanto de la temporalidad con la que el tratamiento antiinflamatorio modifica cada uno de los componentes del asma $^{17}$ como de la elevada y precoz res- 
puesta de los valores de $\mathrm{FE}_{\mathrm{NO}}$ a los corticosteroides inhalados ${ }^{17}$. Apoya esta hipótesis el hecho de que la eosinofilia en esputo se asociase al grado de obstrucción basal dado por el cociente $\mathrm{FEV}_{1} / \mathrm{FVC}$ y que esta correlación que no se mantuviera con los valores de $\mathrm{FE}_{\mathrm{NO}}$.

Recientemente, Vega y $\operatorname{col}^{18}$ han validado al castellano el cuestionario Asthma Control Test, que ha demostrado ser una herramienta útil para cuantificar el control del asma referido al último mes. En nuestro estudio, la puntuación de síntomas se correlacionaba con la pendiente CCR al salino hipertónico, apoyando el valor de esta prueba como indicador de la actividad del asma. Se observó también asociación indirecta de la puntuación de síntomas con los valores de eosinófilos y de $\mathrm{FE}_{\mathrm{No}}$ que, al considerar a los grupos de asmáticos por separado, se incrementaba entre los que no recibían tratamiento antiinflamatorio, para desaparecer en el grupo que tomaba esteroides inhalados. Resalta el hecho de que estas diferencias se observaran también al analizar los eosinófilos en esputo, lo que podría ser también atribuible a los diferentes tiempos con que se modifican los indicadores del asma en respuesta al tratamiento ${ }^{17}$.

Una posible lectura de estos resultados sería que el tiempo de respuesta de la percepción clínica del asma y de los valores de $\mathrm{FE}_{\mathrm{NO}}$ al tratamiento con corticosteroides inhalados, es insuficiente para reducir la actividad de la enfermedad, dada por la eosinofilia en esputo y por la HRB a agonistas indirectos como las soluciones hiperosmolares. Dado que los eosinófilos en esputo no se modifican hasta pasados 6 meses del inicio del tratamiento, sería poco recomendable realizar cambios en éste a pesar de la reducción en los valores de $\mathrm{FE}_{\mathrm{NO}}$ hasta completado dicho periodo.

Parece sin embargo indudable que la cuantificación de $\mathrm{FE}_{\mathrm{NO}}$ en pacientes que no estén recibiendo tratamiento antiinflamatorio puede ser de gran utilidad en el diagnóstico de asma. Así, un paciente con síntomas sugestivos de obstrucción variable de las vías respiratorias y con valores elevados de $\mathrm{FE}_{\mathrm{NO}}$, tendría altas probabilidades de presentar asma, aún en ausencia de una obstrucción bronquial objetiva. Esta aplicación ya se recoge en la nueva Guía Española para el Manejo del Asma (GEMA, 2009), en la que se recomienda realizar una determinación de los valores de $\mathrm{FE}_{\mathrm{NO}}$ previa al establecimiento de un nuevo diagnóstico de asma. Es más dudoso el papel que desempeñan los valores de $\mathrm{FE}_{\mathrm{No}}$ en el seguimiento y la monitorización del tratamiento antiinflamatorio en el asma, ya que los datos a este respecto son controverti$\operatorname{dos}^{25}$. Así, un reciente metaanálisis ${ }^{26}$ tras analizar 356 sujetos concluyó que el seguimiento de los valores de $\mathrm{FE}_{\mathrm{NO}}$ no aportaba beneficios al control del asma respecto a las guías convencionales, salvo que éste se conseguía con menores dosis de tratamiento. Los resultados de nuestro ensayo también pareden estar en consonancia con esta percepción.

En conclusión, la técnica de cuantificación de $\mathrm{FE}_{\mathrm{NO}}$ es rápida, sencilla y aporta datos inmediatos. Los resultados con ella obtenidos se correlacionan aceptablemente con los indicadores de inflamación eosinofílica y de actividad de la enfermedad, en especial en asmáticos que no reciben tratamiento antiinflamatorio. En consecuencia puede ser un indicador útil en el diagnóstico inicial del asma aunque su valor en el seguimiento y control del tratamiento antiasmático a largo plazo es más discutible y necesita nuevas investigaciones.

\section{In memoriam}

Pocos meses antes de completar este trabajo sufrimos la desgracia del fallecimiento de Eduardo Urbiola. Eduardo fue, sin lugar a dudas, una gran persona, excelente patólogo y compañero. Actuó desde el principio como motor y nexo de unión entre los servicios de Anatomía Patológica y Alergología del Hospital Virgen del Camino, en el desarrollo de esta línea de trabajo, de marcadores no invasivos de la actividad inflamatoria en el asma bronquial, que continúa dando sus frutos. Sirvan estas líneas de agradecimiento y recuerdo a su persona. 


\section{Agradecimientos}

Este proyecto ha sido en parte financiado mediante una beca de ayudas a proyectos de investigación del Gobierno de Navarra y mediante el fondo de investigación de alergología en la Fundación Miguel Servet.

\section{BIBLIOGRAFÍA}

1. National, Asthma. Education and Prevention Program. Expert Panel Report 3 (EPR-3): Guidelines for the Diagnosis and Management of Asthma [internet]. Bethesda, MD: National Institutes of Health: National Health, Lung and Blood Institute; 2007. Publication No. 074051 [accessed 2007 Dec 30] http://www.nhlbi.nih.gov/guidelines/asthma/asthgdln.htm.

2. Rabe K, Adachi M, Lai C, Soriano J, Vermeire P, WeISS $\mathrm{K}$ et al. Worlwide severity and control of asthma in children and adults: the global asthma insights and reality surveys. J Allergy Clin Immunol 2004; 114: 40-47.

3. Global. strategy for asthma management and prevention (GINA). In: National Institute of Health, Lung and Blood, editor. NHLBI/WHO workshop report. Washington DC; Revised 2006.

4. Cabañes Higuero N, Fernández Meléndez S, Vega Chicote J, Jiménez LaRA M. Aspectos clínicos del asma: presentación habitual. Formas de presentación atípica y diagnóstico diferencia. En Peláez Hernández A, Dávila González IJ; Tratado de Alergología Vol. 1. ISBN: 97884-8473-576-2; pp 645-654.

5. Fahy JV, Liu J, Wong H, Boushey HA. Cellular and biochemical analysis of induced sputum from asthmatic and from healthy subjects. Am Rev Respir Dis 1993; 147: 1126-1131.

6. Pizzichini E, Pizzichini MM, Efthimiadis A, HarGREAVE FE, Dolovich J. Measurement of inflammatory indices in induced sputum: effects of selection of sputum to minimize salivary contamination. Eur Respir J 1996; 9: 1174-1180.

7. Olaguíbel J, Álvarez M, García B, Tabar A, URBIOLA E. The study of the inflammation of the airway in bronchial asthma by cytological and chemical analysis in the fluid phase of samples taken by sputum induction. An Sist Sanit Navar 1998; 21: 173-186.

8. Álvarez MJ, Olaguíbel JM, García BE, Rodríguez A, Tabar AI, Urbiola E. Airway inflammation in asthma and perennial allergic rhinitis. Relationship with nonspecific bronchial responsiveness and maximal airway narrowing. Allergy 2000; 55: 355-362.
9. Álvarez MJ, Olaguíbel JM, García BE, Tabar AI, URBIola E. Comparison of allergen-induced changes in bronchial hyperresponsiveness and airway inflammation between mildly allergic asthma patients and allergic rhinitis patients. Allergy 2000; 55: 531-539.

10. Green RH, Brightuing CE, McKenna S, Hargadon B, Parker D, Bradding P et al. Asthma exacerbations and sputum eosinophil counts: a randomised controlled trial. Lancet 2002; 360: 1715-1721.

11. Jatakanon A, Lim S, Barnes PJ. Changes in sputum eosinophils predict loss of asthma control. Am J Respir Crit Care Med 2000; 161: 64-72.

12. Gibson PG, Henry RL, Thomas P. Noninvasive assessment of airway inflammation in children: induced sputum, exhaled nitric oxide, and breath condensate. Eur Respir J 2000; 16: 1008-1015.

13. Green RH, Brightling CE, Woltmann G, Parker D, WARDLAW AJ, PAVORD ID. Analysis of induced sputum in adults with asthma: identification of subgroup with isolated sputum neutrophilia and poor response to inhaled corticosteroids. Thorax 2002; 57: 875-879.

14. Baraldi E, Scollo M, Zaramella C, Zanconato S, Zacchello F. A simple flow-driven method for online measurement of exhaled NO starting at the age of 4 to 5 years. Am J Respir Crit Care Med 2000; 162: 1828-1832.

15. de Kluijver J, Evertse CE, Schrumpf JA, Van Der Veen H, Zwinderman AH, Hiemstra PS et al. Asymptomatic worsening of airway inflammation during low-dose allergen exposure in asthma: protection by inhaled steroids. Am J Respir Crit Care Med 2002; 166: 294-300.

16. Jones S, KitTELSON J, CowAn JO. The predictive value of exhaled nitric oxide measurements in assessing changes in asthma control. Am J Respir Crit Care Med 2001; 164: 738-743.

17. Malerba M, Ragnoli B, Raedely A, Tantucci C. Usefulness of exhaled nitric oxide and sputum eosinophils in the long-term control of eosinophilic asthma. Chest 2008; 134: 733739 .

18. Vega J, Badia X, Badiola C, Lopez Viña A, OlaguíBEL JM, PICADO C et al. Covalair Investigator Group. Validation of the Spanish version of the Asthma Control Test (ACT). J Asthma 2007; 44: 867-872.

19. Álvarez Puebla MJ, Olaguíbel Rivera J. Evaluación del componente inflamatorio en las vías respiratorias inferiores. En: Peláez Hernández A, Dávila González IJ; Tratado de Alergología Vol. 1; pp 189-200. 
20. Jatakanon A, Lim S, Kharitonov SA, Chung KF, BARNES PJ. Correlation between exhaled nitric oxide, sputum eoosinophils and methacholine hyperresponsiveness in patientes with mild asthma. Thorax 1998; 53: 91-95.

21. Kharitonov SA, Donnelly LE, Montuschi P, CoRRADI M, Collins JV, Barnes PJ. Dose-dependent onset and cessation of action of inhaled budesonide on exhaled nitric oxide and symptoms in mild asthma. Thorax 2002; 57: 889896.

22. Zacharasiewicz A, Wilson N, Lex C, Erin EM, Li AM, HANSEL T et al. Clinical use of noninvasive measurements of airway inflammation in steroid reduction in children. Am J Respir Crit Care Med 2005; 171: 1077-1082.

23. Holgate S. Adenosin provocation: a new test for allergic type airway inflammation. Am J Respir Crit Care Med 2002; 165: 317-319.
24. Porsbjerg C, Brannan J, Anderson S, Backer V. Relationship between airway responsiveness to mannitol and to methacholine and markers of airway inflammation, peak flow variability and quality of life in asthma patients. Clin Exp Allergy 2008; 38: 43-50.

25. Szefler S, Mitchel $H$, Sorkness C, Gregen $P$, O'Connor G, Morgan WJ Ea. Management of asthma based on exhaled nitric oxide in addition to guideline-based treatment for inner-city adolescents and young adults: a randomised controlled trial. Lancet 2008; 372: 1065-1072.

26. Petsky H, Cates CJ, Li AM, Kynaston JA, Turner $\mathrm{C}, \mathrm{AB} \mathrm{C}$. Tailored interventions based on exhaled nitric oxide versus clinical symptoms for asthma in children and adults. Cochrane Database of Systematic Reviews 2008: Issue 2. Art. No.: CD006340. DOI: 10.1002/14651858. CD006340.pub2 
